\title{
Tout nom et tout pronom : qu'est-ce qui fait la différence?
}

\author{
Catherine Schnedecker \\ Université Marc Bloch Strasbourg 2 \\ EA1339, LiLPa/Scolia \\ cschnede@umb.u-strasbg.fr
}

\section{Introduction}

Si tout, dans ses réalisations en tant que déterminant (tout $+N$, tout le $N$, tous les $N$, tout un $N$ ), a fait l'objet d'une abondante littérature (notamment Anscombre, 2006, Kleiber, à par., Paillard, 2001), il n'en va pas de même, à notre connaissance, pour ses emplois notamment pronominaux (désormais tout $\left._{P}\right)^{1}$ et nominaux $\left(\right.$ tout $\left._{N}\right)$ dont le comportement est plus régulier, donc a priori moins intriguant que celui qu'il a lorsqu'il s'ajoute à un autre déterminant. Pourtant, à y regarder de plus près, ce comportement et cette double appartenance catégorielle ne vont pas sans poser problème, dans la mesure où certains grammairiens réduisent la différence de catégorie à une simple différence d'emploi :

$L$ 'adjectif tout au sens d'entier se prend aussi comme nom et suit alors la syntaxe des noms (...). Ce tout collectif singulier s'emploie aussi comme nom neutre au sens de toute chose (...) mais toujours sans article ce qui le distingue de l'autre nom: tout passe, tout se tait (Martinon, cité par Andersson, 193)

Ce caractère nominal $<\mathrm{de}_{\mathrm{p}} \mathrm{t}_{\mathrm{p}}>$ s'accuse fortement lorsque tout est précédé d'un déterminatif (Le Bidois, §449)

Bon nombre de faits étayent ce point de vue : la définition des deux unités et leurs paraphrases quasiment identiques (1-2) tout $_{P}$ marquant l'idée d'intégralité (TLFi) et tout ${ }_{N}$ celle - qui n'est guère plus éclairante de totalité ; leur usage qui se croise à maints endroits: dans leur complémentation en de (3-4), leurs emplois attributifs liés aux constructions ce n'est pas (le) tout (de+inf) +que+subj (5-9) et, enfin, leur rôle dit récapitulatif par les dictionnaires, qu'illustrent (10) et (11):

1) Tout $\approx$ l'ensemble des choses dont il est question (soit toutes choses)

2) Le tout $\approx$ l'ensemble dont les éléments viennent d'être désignés; l'ensemble des choses dont on parle, l'unité qu'elles forment ; l'ensemble de toutes choses (Petit Robert 2008)

3) Il me semble bien que la sympathie artistique suffit, que le principal est de faire des peintures vivantes, et que c'est même le tout de l'art, (...) (Lemaître, Contemp., 1885)

4) Il t'donne un bon conseil et tu l'envoies ch... T'as tout d'la vache (Dorgelès, Croix de bois, 1919)

5) Ce n'est pas tout d'avoir la foi, que d'avoir la foi, il faut faire de bonnes auvres (Ac. 1798-1878)

6) Ce n'est pas tout que la France soit sauvée par les armes, il faut qu'elle trouve son équilibre et sa reconstitution (Barrès, 1919)

7) Au fond, voyez-vous, le chagrin, c'est comme le ver solitaire: le tout, c'est de le faire sortir (Pagnol, Fanny, 1932)

8) Vial aura besoin de papa, l'hiver prochain, si le ministère ne tombe pas, parce que papa est camarade de collège avec le ministre... Le tout est que le ministère ne tombe pas avant que les Quatre-Quartiers aient mis Vial à la direction de leurs ateliers (Colette, Naiss. jour, 1928)

9) Je vous comprends, mon fils; mais ce n'est pas le tout de couper, il faut recoudre (Dumas père, Henri III, 1829)

10) Affronter une fois de plus l'odeur de mangeaille, le service bruyant, la promiscuité de l'éternelle popote, écouter avec un sourire complaisant les palabres quotidiennes sur les projets de l'Allemagne, les calculs sur la durée de la guerre, l'explication des sous-entendus du communiqué... -le tout, assaisonné de taquineries rituelles, de souvenirs du front (Martin du Gard, 1940, TLFi). 
11) Ce millionnaire, le père Labille, qui avouait avoir tout eu, fortune, santé, bonheur de ménage (Goncourt, Journal, 1860)

L'objectif de ce travail est de double puisqu'il s'agit de montrer, au contraire, principalement sur la base d'exemples empruntés à Frantext, que la partition nom vs pronom est bien fondée au plan morphosyntaxique et qu'elle trouve des échos sémantiques dans le mode de donation particulier des « ensembles » ainsi visés. On espère ainsi fournir des éléments de connaissance sur un pronom resté en marge des études, continuer, par la comparaison nom/pronom, à défricher le terrain des $\mathrm{N}$ de «totalité » (voir Schnedecker, à par. a et b, Lammert, à par.) et, secondairement, amorcer un tout début de réflexion sur une complémentarité entre les formes en tout, qu'elles soient déterminant, pronom ou nom.

\section{Tout $_{P}$ vs tout to $_{N}$ une différence de catégorie indéniable}

\subsection{Différences morpho-syntaxiques}

La première série de clivages qui sépare les deux formes est à caractère morpho-syntaxique. Le plus évident tient à la morphologie : tout $_{P}$ est invariable alors que tout $_{N}$ varie en nombre $(12-13)^{2}$. On en verra les incidences sur le mode de donation référentiel.

12) (...) et cette union accidentelle devient si intime, que les deux touts n'en forment plus qu'un seul. (Bonnet, corps organisés, 1762)

13) Que le Corps des Représentans étant essentiellement un, ne peut pas se diviser de manière à former plusieurs touts ou chambres exerçant un veto l'un sur l'autre, (Sieyès, Euvres, 1791)

En second lieu, tout $_{P}$ est bien irréductiblement un pronom, et, qui plus est, un pronom qui n'a plus rien à voir avec le $\mathrm{N}$ ainsi que le prouvent trois arguments. Premièrement, il se démarque d'unités comme plusieurs ou certains dont les emplois pronominaux ne sont, d'une certaine façon, que des « déterminants sans nom » dans la terminologie de Corblin, c'est-à-dire des unités dont le $\mathrm{N}$ se laisse aisément récupérer dans le cotexte (14) ou facilement récupérer via le simple recopiage (15) ou la construction en de $N(16)$ :

14) Les socialistes sont désorientés; plusieurs sont même déprimés

15) Les socialistes sont désorientés; plusieurs [socialistes] sont même déprimés

16) Les socialistes sont désorientés ; plusieurs, de socialistes sont même déprimés

Or, tout ne présente aucune de ces propriétés : il n'est en effet pas question de procéder à la récupération d'un $\mathrm{N}$ comme c'était le cas ci-dessus :

\section{7)*Tout, (de produit+de chose) est cher}

Par ailleurs, force est de constater que les paraphrases à base nominale proposées par les dictionnaires sont parfois difficiles à appliquer et qu'elles sont, d'autre part, inégales dans leur rendement :

18) Tout est cher $\approx$ tout produit/toute chose est chèr(e)

19) tout va bien \#? toute chose va bien

Enfin, tout $_{P}$, contrairement à d'autres pronoms, et surtout, contrairement à tout $_{N}$, est réfractaire aux expansions adjectives ou relatives (20 vs 21-22), ne tolérant que les expansions adnominales illustrées en (25-26) qui n'ont toutefois n'ont pas la même valeur: (25) est paraphrasé par Andersson par "être complètement, avoir toutes les qualités, ressembler tout à fait à » alors que (27) et (29) équivalent à la totalitéll'ensemble de en quoi ils rejoignent certains emplois de tout $_{N}$ :

20)*Paul aime tout (beau+qui est beau) 
21) Dominant de pensée et de beauté ce tout colossal (Londres) je vois Westminster Abbey, le Parlement (Sandfeld in Andersson, 237)

22) (...), enfin les deux noires et massives tours avec leurs auvents d'ardoise, parties harmonieuses d'un tout magnifique, superposées en cinq étages gigantesques, (...) (Hugo, Notre-Dame, 1832)

23) De tous ces êtres diversement combinés dans des proportions très variées, il résulte des corps, des touts physiques ou moraux dont les propriétés et les égalités sont essentiellement différentes, (Holbach d', Système de la nature, 1770)

24) Si, en effet, ignorer l'histoire revient à nier le réel, c'est encore s'éloigner du réel que de considérer l'histoire comme un tout qui se suffit à lui-même. (Camus, Homme révolté, 1951)

25) Elle a tout d'une grande (slogan publicitaire) $\approx$ elle a toutes les qualités d'une grande

26) Elle a tout de Sainte Thérèse (in Andersson, 197) ₹ elle a toutes les qualités de Sainte Thérèse

27) Je regrette tout de ce beau petit passé, je regrette tout de vous, garçons, même vos maladies (Duhamel in Andersson, ibid.)

28) Je regrette (la totalité +l'ensemble) de ce beau petit passé

29) (...) la lumière la plus ancienne, celle qui contient le tout de l'enfance, s'inscrit encore dans le même paradoxe que je poursuis (...) (Roubaud, Boucle, 1993)

30) (...) la lumière la plus ancienne, celle qui contient (la totalité +l'ensemble) de l'enfance

Le troisième type de clivage entre tout $_{N / P}$ concerne leur syntaxe. Tous deux occupent sans réserve toutes les fonctions syntaxiques (sujet (31-32), COD (33-34), COI (35-36) ou CC (37-38)) mais pas les mêmes places dans l'énoncé, seul tout $t_{P}$ ayant la capacité de s'insérer entre auxiliaire et participe passé (39 vs 40) ou entre le modal et son infinitif (41 vs 42):

31) Tout va bien

32) Le pavé est aussi en entier de mosaïque. Le tout a été si bien joint que, quoique le pavé soit enfoncé dans certains endroits et fort relevé dans d'autres, aucune petite pièce ne s'est démontée (...) (d'Ormesson, Douane de mer, 1993)

33) Paul aime tout

34) Isy s'était chargé d'installer le tout et avait même fait disparaître les plastiques d'emballage. (Jonquet, Orpailleurs, 1993)

35) Paul a pensé à tout

36) On a affaire à un tout concerté (Roubaud, Poésie, 2000)

37) Paul a des compétences en tout/dans tout

38) Dans les constructions modernes, la caisse et le châssis sont souvent confondus en un tout rigide. (Bailleul, Matériel roulant des chemins de fer, 1951)

39) J'ai tout mangélj'ai mangé tout

40) *J'ai le tout mangélJ'ai mangé le tout

41) Je veux tout manger/je veux manger tout

42) *Je veux le tout manger/Je veux manger le tout

Par ailleurs, le parallélisme entre les constructions attributives évoqué en introduction ne vaut en réalité que pour les constructions négatives et ne connaît pas d'équivalent pour le pronom avec les formes assertives (46 vs 44, 47 vs 44) :

43) Ce n'est pas tout (que) d'avoir la foi, il faut faire de bonnes æuvres (Ac, TLFi)

44) Ce n'est pas tout que la France soit sauvée par les armes, il faut qu'elle trouve son équilibre et sa reconstitution (Barrès, TLFi)

45) Ce n'est pas le tout de couper, il faut recoudre (Dumas, TLFi)

46) *C'est tout (que) d'avoir la foi

47) *C'est tout que la France soit sauvée par les armes

Cette différence de construction en révèle d'ailleurs une autre : tout $_{N}$ se laisse disloquer, pas tout $_{P}$ : 


\subsection{Différences discursives}

Les derniers points de rupture entre les deux tout sont à caractère discursif. D'abord, s'ils ont vocation à la récapitulation comme signalé plus haut, les modalités de celle-ci diffèrent. Tout $_{P}$ en effet, opère aussi bien en amont (50) qu'en aval (49) à la différence de $t_{0} t_{N}$, plus spécialisé ${ }^{3}$, lorsqu'il est précédé de l'article défini du moins, pour la reprise anaphorique (51), la cataphore étant plus sporadique (i.e. moins répandue et limitée à quelques auteurs, comme, p.e. C. Simon (52)) :

49) Habitués à dévorer, engloutir tout à l'aveugle (animaux, bois, pierre, n'importe), cette fois, chose admirable! ils s'abstiennent. (Pontalis, Fenêtres, 2000)

50) (...) à cause des haut-parleurs, c'est la musique qui vous attire, et puis, plus près, voilà les paroles, dialogues et bruitages, l'histoire. On entend tout. (Bon, Mécanique, 2001)

51) Une table et quatre chaises, un cosy et une armoire, un buffet et deux tabourets- le tout dans cet excellent bois blanc qui pompe si bien des litres de brou de noix (Bazin, petit cheval, 1950)

52) (...) faisant entendre de quart d'heure en quart d'heure un tintement aigrelet, dérisoire et mutin, le tout - angelots, sphinges, guirlandes, urne, marbre et bronze - ayant quelque chose de funéraire et de futile, (...) (Simon, Herbe, 1958)

Cela explique aussi que tout $_{N}$, du fait de sa position au terme d'une énumération, ne fasse que rarement l'objet d'une reprise anaphorique ${ }^{4}$, sauf en contexte de recettes de cuisine, alors que tout $_{P}$ l'est $^{\prime}$ principalement par le biais de l'anaphore démonstrative (55-56), - la définie passant plus difficilement ou autrement au vu de (57-58) -, qui résonne alors comme « mentionnelle » plutôt que comme référentielle ${ }^{5}$ :

53) vous les faites ensuite mariner avec sel, poivre, basilic, muscade, persil, ciboule, échalotes, une petite gousse d'ail et deux champignons, le tout haché bien menu, et un verre d'huile d'olive. Lorsque le tout a bien mariné pendant soixante minutes, vous arrangez vos filets dans une grande casserole, (Grandes heures de la cuisine française, 1964)

54) il y a quatre professeurs de mathématiques, il faudrait que leur service, au lieu d'être divisé plus ou moins arbitrairement en quatre lots isolés, constitue un tout, et que ce tout soit placé sous l'autorité de l'un des quatre professeurs (Capelle, École de demain, 1966)

55) Je sais tout, mon cher ami. Ce tout n'est pas grand'chose (in Andersson, 204)

56) Il valait mieux qu'il sût tout, puisque ce tout n'était rien (in Andersson, ibid.)

57) Je sais tout, mon cher ami. ?Le tout n'est pas grand'chose

58) Il valait mieux qu'il sût tout, \# puisque le tout $n$ 'était rien

Ensuite, les emplois résomptifs de tout $_{N}$ apparaissent principalement dans des segments discursifs « détachés », plus ou moins fortement selon les cas (points (59) ou virgules (60)), et dénués de noyau verbal tensé (62), qui constituent des formes d' " appendices », de fragments secondaires apportant des précisions sur l'énoncé marqué comme principal :

59) (...) : protéger d'une main la flamme, l'approcher des vieux journaux, les allumer en différents endroits, avec le petit bois déjà disposé dessus. Le tout, très vite, évidemment. (Schreiber, silence, 1996) 60) (...) les rapporter garnis du plus grand nombre possible de litrons, tant de rouge que de blanc. Une fois cette mission accomplie, il ne restait plus qu'à les boire, le tout, bien entendu, à l'insu de l'encadrement. (Rolin, Organisation, 1996)

61) et de toutes les couleurs, de chiffons innommables, de poubelles en osier remplies de serpillières définitivement trempées, de deux sacs de linge sale débordants, le tout sableux et collant. (Brisac, Weekend de chasse, 1996)

62) Une assiette de mozzarella et tomates, avec câpres, anchois et olives noires. Un plat de spaghetti aux clovisses. Un tiramissu. Le tout arrosé d'un bandol du domaine de Pibarnon. (Izzo, Total Khéops, 1995) 
Or, ce type d'emploi est prohibé pour tout $t_{P}$ sauf à modifier considérablement son interprétation (65) :

63)?(...) : protéger d'une main la flamme, l'approcher des vieux journaux, les allumer en différents endroits, avec le petit bois déjà disposé dessus. Tout, très vite, évidemment.

64) *(...) les rapporter garnis du plus grand nombre possible de litrons, tant de rouge que de blanc. Une fois cette mission accomplie, il ne restait plus qu'à les boire, tout, bien entendu, à l'insu de l'encadrement.

65) \# et de toutes les couleurs, de chiffons innommables, de poubelles en osier remplies de serpillières définitivement trempées, de deux sacs de linge sale débordants, tout sableux et collant.

66) *Une assiette de mozzarella et tomates, avec câpres, anchois et olives noires. Un plat de spaghetti aux clovisses. Un tiramissu. Tout arrosé d'un bandol du domaine de Pibarnon.

Ces premières observations montrent donc d'indéniables différences morpho-syntaxiques et discursives entre les deux tout et invite à examiner leurs divergences au plan sémantico-référentiel qui, comme on va le voir, révèlent deux manières distinctes de conceptualiser des « ensembles ».

\section{Tout $_{P} /$ tout $_{N}$ ou l'expression d' " ensembles » différenciée}

La paraphrase des dictionnaires (cf. supra (1-2)) est parlante : out $_{P / N}$ renvoient à des «ensembles ${ }^{6}$ que nous étudierons d'abord du point de vue de leurs constituants puis du type d'entité complexe qu'ils « forment » à leur tour.

\subsection{Les « constituants » des deux ensembles}

\subsubsection{Points communs}

Les entités constitutives des deux tout présentent trois points communs. D'abord elles sont plurielles ( $c f$. les paraphrases des dictionnaires), ce que démontrent les enchaînements suivants, plus acceptables quand l'antécédent est un ensemble (68) que lorsqu'il est une unité $(67)^{7}$ :

67) Lucie a acheté une robe. *Elle a tout payé cash $/{ }^{*}$ Elle a payé cash le tout ${ }^{8}$

68) Pendant les soldes, Lucie a acheté deux robes, trois paires de chaussures, un manteau, deux sacs à main, une paire de lunettes de soleil. Elle a tout payé cash/Elle a payé le tout cash

Elles sont ensuite hétérogènes entre elles ${ }^{9}$, ce qui apparaît, d'une part, à travers les emplois résomptifs des deux tout, qui enchaînent généralement sur une série de $\mathrm{SN}$ visant des unités éclectiques, relevant de catégories référentielles bien distinctes les unes des autres ( $c f .61-62$ et 69), avec pour tout $_{N}$ la possibilité, rare au demeurant, de reprendre des SN pluriel et donc de viser des entités homogènes (70-71) :

69) Peut-être encore une fois, une seule... Ne pas pouvoir se souvenir de tout, la tendresse, les mots vagues, ou bien de chaque signe concret manifestant cette tendresse. (Ernaux, Se perdre, 2001)

Le bidet, la carriole, la laitière et les pots de laits, tout culbute. (La Fontaine, in Le Bidois, §446)

70) le "tutu» proprement dit, composé de quatre petites jupes superposées dont les diamètres vont progressivement en croissant de 6 cm vers le haut, le tout monté sur un empiècement taillé en forme et prenant la taille très serrée ainsi que le haut des hanches; une culotte courte et à volants appelée trousse, (...) (Bourgat, Technique de la danse, 1959)

71) L'on calculera ensuite, un par un, les mouvements élémentaires de ces éléments, l'on additionnera le tout et l'on pourra obtenir un résultat macroscopique fidèle. (David, Cybernétique et l'humain, 1965)

Cela explique en partie pourquoi les lexicographes privilégient le $\mathrm{N}$ de choses dans les paraphrases pour désigner les entités plurielles ainsi visées. Le $\mathrm{N}$ de chose n'est pas, comme l'a démontré Kleiber (1994), 
une dénomination - autrement dit chose n'est pas le $\mathrm{N}$ des choses - et il a la capacité de renvoyer à des catégories ontologiques aussi diversifiées que les entités concrètes, les actions, les événements, etc.

Enfin, les constituants des deux tout sont hétérogènes par rapport à leur tout (qui n'est pas de même nature/catégorie que les « parties »).

\subsubsection{Différences}

Cela étant dit, les entités constitutives des deux tout se distinguent par trois aspects au moins. Premièrement, celles de tout $_{N}$ peuvent être dénommées par des N comme partie (72), pièce (74) ou élément (73), ce qui est impossible pour tout $_{P}(76)$ :

72) il définit par exemple les 21 ou 29 «civilisations » qu'il a, un peu arbitrairement, découpées dans le tissu de l'histoire comme "des ensembles formant un tout dont toutes les parties sont en cohésion réciproque et s'affectent mutuellement (Marrou, connaissance historique, 1954)

73) les autres ne sont que des éléments d'un tout, qu'il y a, certes, quelque arbitraire à scinder (Tiers monde, dir. de G. Balandier, 1956)

74) C'est le texte comme ensemble de phrases (le terme "phrase " étant plus adéquat qu'« énoncé » dès qu'il s'agit d'une pièce au sein d'un tout cohérent). (Hagège, Homme de paroles, 1985)

75) une partie du tout vs un *(morceau+bout+portion + part $) d u$ tout

76) *X est une (partie+pièce+élément) de tout

C'est ce que confirme notamment l'association, dans l'usage, des unités lexicales tout et partie (77) au point même de constituer en une sorte de déterminant complexe tout ou partie de $N(78)^{10}$ :

77) Le tout est égal à la somme de ses parties

78) Vendre tout ou partie de son bien

Deuxièmement, les entités de tout $_{P}$ semblent dotées d'un degré d'hétérogénéité supérieur à celle de tout $_{N}$. En effet, dans les emplois résomptifs de tout $_{N}$, les entités de départ, si elles relèvent de catégories différentes, s'inscrivent néanmoins, le plus souvent, toutes dans la même catégorie hyperonyme (objets concrets (61-62), événements (59-60), maladies (79), qualités (80), actes de langage (81) ...), ce qui n'est pas systématique avec tout $_{P}$ (cf. supra (69) et infra (82-83)) :

79) j'attraperai tours de reins, torticolis, courbatures et lumbagos et je vous dédierai le tout, belle cruelle, féroce blonde, couturière sans coeur! (Fallet, Triporteur, 1951)

80) Mais j'aime Popeline. Elle est si belle, si grande et si douce que le tout en fait une adorable jeune fille. (Fallet, Triporteur, 1951)

81) (...) mais le texte récité est lui-même plein de force, avec ses impératifs multiples, ses appels pressants, ses promesses magnifiques ou ses menaces terribles, le tout en une prose rythmée, renforcée de particules d'affirmation, (...) (Philosophie, religion, dir. Gaston Berger, 1957)

82) Au beau milieu de la ville on vous offre, tout simplement, une colline, le plan de la ville, puis, sur l'atlas, la carte d'Écosse, sa belle allure, son élégance, son panache fractal, avec sa côte infiniment dentelée d'îles à l'ouest. Je rêvais de tout en connaître, (...) (Roubaud, Poésie, 2000)

83) Les roses, les retrouvailles, la soirée : tout était là, écrit noir sur blanc, et une multitude de détails que je connaissais de sa vie me sautaient à présent aux yeux, (Bouillier, Invité mystère, 2004)

Troisièmement, les entités de tout $_{P}$ ne sont pas nécessairement regroupées au plan spatio-temporel, autrement dit, elles ne sont pas nécessairement liées par une contrainte de proximité, ce que prouvent (8485 ) où les actions dénotées par les énoncés ne peuvent avoir lieu simultanément :

84) (...) tout va bien : je dors je mange je ne fais rien ou presque et je ne m'ennuie même plus. J'ai reçu des foules de lettres ce qui me donne une occupation rien qu'à les classer ; (...) (Roubaud, Bibliothèque de Warburg, 2002) 
85) tout est infiniment difficile, corriger des copies, faire quelques mots de russe (à quoi cela sert-il). (Ernaux, Se perdre, 2001)

Par contraste, les prédicats appliqués à tout $_{N}$ montrent que les entités ainsi visées sont ancrées dans un même espace-temps (86-88) :

86) Derrière la maison, s'étendait une pelouse carrée, faisant jaillir en son milieu un jet d'eau autour duquel rayonnaient des palmiers nains et des manguiers. Le tout entouré d'une haie d'hibiscus élevée et taillée, compacte comme un mur. (Zobel, Rue Cases-Nègres, 1950)

87) Il ferme le calepin, glisse le crayon dans l'étui cousu à la couverture, replace le tout dans sa poche, (Genevoix, Ceux de 14, 1950)

88) Il ajouta : "je suis venu en vélo, j'aurais fourré les débris dans un sac, attaché une pierre au sac et balancé le tout dans la rivière ; je me serais bien débrouillé tout seul. (Beauvoir, Les Mandarins, 1954)

\subsection{Caractéristiques des « ensembles » visés par tout $t_{P / N}$}

De là vient que les ensembles conceptualisés par tout $_{N / P}$ ne présentent pas les mêmes propriétés.

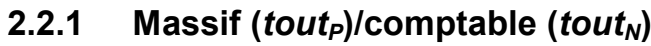

La première grande " opposition » entre ces deux types d' « ensembles » tient à ce que tout $_{P}$ est massif, tout $_{N}$ comptable. C'est ce que montre, premièrement, leur comportement à l'égard de verbes tels que compter, inventorier, ou recenser ( $c f$. Galmiche, 1988) dénotant les activités de dénombrement, recensement. A priori comme le suggèrent (86) et (89), les deux formes sont compatibles avec ces verbes. Mais l'interprétation des énoncés diffère. En effet, (86) se paraphrase par (87), ce qui montre que les entités du tout en question ont fait l'objet d'une opération de dénombrement, sans pour autant permettre une quantification (d'où l'impossibilité de (88)), à la différence de ce qu'autorise tout $_{N}(89-90)$ :

86) J'ai tout (compté+ inventorié+ rencensé)

87) Tout a fait l'objet d'un (décompte+inventaire+recensement)

88) *Tout (se chiffre + s'élève +équivaut) à $n €$

89) J'ai (compté+ inventorié+ rencensé) sept touts

90) Le tout (se chiffre $+s$ 'élève+équivaut) à $150 €$

Deuxièmement, tout $_{P}$ est parfaitement compatible avec les unités - déterminatives ou adverbiales utilisées avec les $\mathrm{N}$ massifs : le partitif $d e(91-92)^{11}$ ou le quantifieur un peu de (93). Il en a également le mode de reprise par le pronom en (92 et 95) (et non par le pronom le) alors que tout $_{N}$, de son côté, est déterminé par les quantifieurs des $\mathrm{N}$ comptables ( $c f$. supra, point 1.1. et note 2 ) :

91) On trouve de tout à Paris, même de la province du Gard (Du Gard, in Sandfeld, 411)

92) Des petits-fours... des canapés...des pains surprise... des fruits... des... ffff... je ne peux pas te dire. Il y avait de tout, de tout. Je t'en donne? Fais-moi plaisir. Il faut te prier. (Mréjen, Eau sauvage, 2004)

93) Il y avait à terre un peu de sa raison, un peu de sa mémoire. Un peu de sa fierté et de son courage. Un peu de son sang et de sa chair. Un peu de tout d'elle-même, si bien qu'elle ne parvenait plus à se ressaisir. (Lanzmann, Horde d'or, 1994)

94) Que nous conseillez-vous? Un menu pour les bons clients? Ah! Préparez-nous l'assiette du chef, ce que vous voulez, je vous fais confiance. Avec un peu de tout, très bien. (Mréjen, Eau sauvage, 2004)

95) On tient chacun le verre d'une main comme si j'avais trois ans. Je bois tout sans en renverser. Eh ben dis donc! tu avais soif.) (Mréjen, Agrume, 2001) vs*Je bois tout sans le renverser.

\subsection{2 « Tout » non structuré (tout $\left.t_{P}\right)$ vs structuré (tout $\left.{ }_{N}\right)$}

Le second clivage entre les deux tout tient aux propriétés de l'«ensemble visé ». Conséquence de son caractère massif, celui de tout $_{P}$ n'est pas constitué de "parties», ce qui empêche le pronom de se 
construire avec les « relateurs de tout à partie » étudiés par Tamba (1994) : avoir, être une partie de, se composer de, être formé de, comporter, etc. (96) :

96) Tout a $x^{12}$

D'ailleurs, ces relateurs, quand ils apparaissent aux côtés de tout, le font dans des emplois qui n'ont rien à voir avec l'expression d'une relation de type «partie-tout ». En effet, soit ils sont intransitifs (97-98), soit l'expression converse qui permettrait l'établissement de ce rapport est bloquée (99-101) :

97) (...) la série annuelle se divise dans nos climats en deux saisons marquées ; dans l'une, principe de vie, tout se compose, s'augmente, s'anime, se développe ; dans l'autre, époque d'altération et de dissolution, tout se repose, s'arrête, se corrompt, se détruit. (Senancour, Rêveries, 1802)

98) Et tout se compose bien, grâce à la présence suscitée par la jalousie de la belle fille (Proust, Temps retrouvé, 1922)

99) Et puis il est prisonnier de son chant. Il est une unité de son ouvre, un charme continu du récit que l'artiste doit sauvegarder. Il faut que tout se compose. (...) (Guéhenno, Jean-Jacques, 1948)

100) Il ne faut jamais oublier, en tout cas, que tout se compose de matières sulfureuses et mercurielles, selon une définition due à un alchimiste grec, (...) (Caron \& Hutin, Alchimistes, 1959)

101) *(des) matières sulfureuses et mercurielles sont une partie de tout

De même, les verbes exprimant la désagrégation sont très peu nombreux (se désagréger (105): 1 occurrence dans toute la base Frantext, se désassembler : 3, se défaire (102): 17, se décomposer (103): 10) et se construisent toujours sans compléments, preuve qu'il est difficile de conceptualiser le résultat (parties, pièces, ou autres) du processus :

102) -mais ma fièvre se calme et je ne la reconnais pas et tout se défait. (Alain-Fournier, Corresp., 1914) 103) en vain le demi-tour pour rejoindre, en arrière, les zones où l'air vous soutenait, solide et plein comme un pilier. Mais il n'est plus de pilier. Tout se décompose, et l'on glisse dans un délabrement universel (Saint-Exupéry, Terre des hommes, 1939)

104) (Ronsard: " Mon corps s'en va descendre où tout se désassemble ».) (Roubaud, Poésie, 2000).

105) Notre vertu, Seigneur, est toute faite de résistance; mais autour de nous maintenant tout cède, tout se désagrège, et nous ne sentons plus nos courages. (Gide, Voyage d'Urien, 1893)

Il en va différemment de tout $_{N}$ qui est compatible avec les relateurs se composer de, réunir, être un assemblage de, ce qui prouve qu'il est constitué de plusieurs parties (cf. supra) et que son référent est «compositionnel» :

106) Un tout (se compose+réunit +est un assemblage de) vs *(a+comprend + comporte) ...

Ces relateurs signalent ( $c f$. Tamba, art. cit.), par opposition à comporter, comprendre, que le tout implique l'exhaustivité des parties. Par ailleurs, tout n'admet pas la construction en pour +infinitif montrant que la partie a une fonction dont le «tout» a le contrôle ${ }^{13}$ (107), comme l'illustre (108):

107) Un couteau se compose d'une lame pour couper

108) ? Un tout se compose de parties pour ...

En outre, du fait que, comme montré plus haut, les parties en cause ne sont pas nécessairement de même catégorie, le «tout» ainsi visé peut être dit composite. 
Enfin, compte tenu de la nature de ses parties ( $c f$. supra), cet «ensemble» est doté d'une certaine structure, ce que confirme la présence, dans l'environnement de tout, de prédicats verbaux (former, composer,... (109-110)), ou adjectivaux (111-112) dénotant cette propriété :

109) Multitude bigarrée d'éléments microscopiques, multitude assez grande pour envelopper la terre, et pourtant multitude assez apparentée et sélectionnée pour former un tout structurellement et génétiquement solidaire (Teilhard de Chardin, Phénomène humain, 1955)

110) Les études qui suivent composent un tout. (Perroux, Économie du XXe siècle, 1964)

111) (...) ainsi les grandes religions, (....), qui combinent en un tout véritablement organisé, sentiments, croyances, principes de morale, liturgie, organisation sociale... (Marrou, connaissance historique, 1954) 112) L'inégalité d'un ensemble, considéré comme un tout structuré, à l'égard d'un autre ensemble économique considéré comme tout structuré n'existe pas. (Perroux, Économie du XX siècle, 1964)

\subsection{Des ensembles différemment « bornés »}

La dernière propriété des ensembles visés par les deux tout tient à leur caractère borné, la nature des bornes, si l'on peut dire, variant selon le cas.

Dans le cas de tout $_{N}$, ces bornes sont intrinsèques au sens où elles tiennent à sa nature nominale, d'une part, le nom construisant, comme on sait, des entités délimitées ( $c f$. Langacker) et, d'autre part, à la présence du déterminant.

En revanche, de nombreux arguments étayent l'idée que le référent de tout $_{P}$ serait a priori non borné. Premièrement, il est strictement pronominal ou, pour dire les choses autrement, totalement affranchi du N, donc de toute classe susceptible d'en délimiter le référent; deuxièmement, il est massif et, enfin, les entités de son « ensemble » sont non seulement hétérogènes, mais elles peuvent être également éparses, ce qui rend difficile toute délimitation a priori. Ce à quoi font écho d'ailleurs certaines grammaires en disant que «tout marque la totalité des choses (...) dans une généralité illimitée » (Sandfeld, 411). En outre, tout $_{P}$ englobe non seulement des occurrences factuelles mais également - ce qui le rapproche, d'une certaine façon (mais $c f$. infra), de tout $N$ ( $c f$. Kleiber, à par.) - des occurrences potentielles ou contrefactuelles, ce que prouvent ses emplois fréquents en contexte "générique»(113-116), ou ses affinités avec les auxiliaires de mode $(113,117,119)$, le conditionnel (118) ou les énoncés hypothétiques (119) qui font qu'on peut considérer tout $_{P}$ comme renvoyant à un « ensemble» ouvert :

113) «Le poète doit pouvoir parler de tout en toute liberté ; essayez donc un peu, mes amis, pour voir que vous n'êtes pas libres. " (Roubaud, Poésie, 2000)

114) Tout est bien qui finit bien

115) Tout vient à point pour qui sait attendre

116) Le temps révèle tout : c'est un bavard qui parle sans être interrogé. (Euripide)

117) Enfin, je sais que tout peut arriver, qu'il puisse ne pas venir, un accident. (Ernaux, Se perdre, 2001)

118) la veille j'aurais été prêt à tout pour l'anéantir. (Guibert, Mausolée des amants, 2001)

119) (si on devait tenir compte de tout, il n'y aurait plus de coüncidences; les coüncidences demandent de sérieuses restrictions dans la prise en compte des touts). (Roubaud, Poésie, 2000)

Il en résulte d'ailleurs une conséquence importante qui est que le référent du pronom paraît, d'une certaine façon, plus abstrait que ne l'est celui de $t_{0 u} t_{N}$ puisque, d'après les observations précédentes et pour reprendre ici les critères de Galmiche \& Kleiber (1996), il n'a pas nécessairement de forme ou de matière, ni n'est doté des propriétés perceptuelles, comme le montrent a contrario les énoncés (120) et (121), moins problématiques - disons moins contraignants ( $c$. infra) - dans leur version virtuelle que dans leur version « réelle » (122-123) :

120) dans le marais, et dans le village à deux rues, une en haut, l'autre en bas, chacun sait tout de ce qui se passe à vingt kilomètres. Quand bien même on voudrait se cacher, tout est perceptible à l'ail nu à dix kilomètres dans toutes les directions (Bon, Mécanique, 2001) 
121) Je me défendrai toujours d'être un photographe : cette attraction me fait peur, il me semble qu'elle peut vite tourner à la folie, car tout est photographiable, tout est intéressant à photographier (Guibert, Mausolée des amants, 2001)

122) \# tout est perçu à l'œil nu à dix kilomètres dans toutes les directions

123) \# car tout est photographié

Or, de manière un peu paradoxale, tout $_{P}$ est néanmoins appréhendé comme une « totalité », donc comme doté de bornes, si l'on admet, avec Kleiber (1998), que :

Tout, parce qu'il indique la totalité, nécessite d'une part, un domaine de quantification borné et d'autre part une structuration interne partitive de ce domaine. La première exigence s'explique aisément: je ne puis parler de totalité que pour une entité qui a des limites. L'absence de frontière nous prive en effet du moyen de calculer la totalité. (Kleiber, 1998, 90) ${ }^{14}$

Deux des tests proposés par Kleiber (à par.) étayent d'ailleurs cette idée pour ce qui concerne out $_{P}$ : la compatibilité des marques de totalité avec les constructions signalant les exceptions (124), l'approximation (125) ainsi que l'incongruité de questions identificatoires à leur suite (126) :

124) Comme j'ai presque tout oublié - sauf la disposition du lit par rapport à la fenêtre et à la télévision - de celle où j'étais dans ce même hôtel Amigo, en février 1986, avec Z., (...) (Ernaux, usage de la photo, 2005)

125) Dans ces jours passés avec T., partageant pratiquement tout du déroulement des jours et des nuits, les repas, les promenades et le sommeil, jamais je ne me suis senti aussi étouffé par le manque de tendresse (...) (Guibert, Mausolée des amants, 2001)

126) il supportait tout sans presque protester (...) (Roubaud, Poésie, 2000)

-*Tout quoi?

A quoi s'ajoutent d'autres arguments, comme l'emploi de tout $_{P}$ avec les formes de bornage qu'expriment un $\mathrm{N}$ comme fin (127), les verbes commencer et finir (128) ou encore les constructions comparatives, qui supposent certaines formes de délimitation (129-130), tout comme les locutions prépositives localisatrices, donc « bornantes », au dessus-de (131) et par desssus (132):

127) Qui chercherait-on inlassablement à combler en sachant qu'on n'y arrivera pas ? Et que, si on y parvenait, ce serait la fin de tout. (Pontalis, Fenêtres, 2000)

128) C'était l'été. J'avais dix-sept ans. Pas de parents, pas de connaissances. Tout changeait. Tout finissait. Tout commençait. J'avais fini le lycée, j'avais quitté Saint-Germain-en-Laye. (Roubaud, Poésie, 2000)

129) quand j'entends cela, ces appréciations élogieuses, impression qu'il s'agit d'une autre femme, plus talentueuse, plus tout, que moi : une sorte d'idéal, (Ernaux, Se perdre, 2001)

130) Ce silence total qui sera le sien, pour toujours ou pour des années, me mènera plus sûrement que tout à l'oubli, seule consolation. (Ernaux, Se perdre, 2001)

131) juillet-aồt 88, le déjeuner avec Christiane B., Annie M. ou la rédaction de mon article pour le Dictionnaire de Garcin, je sens comme le temps a passé. Au-dessous de tout, même du souvenir. (Ernaux, Se perdre, 2001)

132) quand elle s'éveillait à son tour, il lui disait : "Ce que j'aime par-dessus tout ce sont tes yeux; tes beaux yeux bruns. » Elle souriait et ne disait rien. (Roubaud, Bibliothèque de Warburg, 2002)

Enfin, à côté des énoncés à caractère générique ou contrefactuel vus plus haut, tout $_{P}$ apparaît également dans des énoncés instituant des bornes ${ }^{15}$, par l'intermédiaire des temps grammaticaux, passé simple (133) ou composé (134-135), - ce qui le distingue de tout $+N$ - ainsi que par des adverbiaux ou des circonstants servant à délimiter des domaines spatiaux (136), temporels ${ }^{16}$ (137-140) ou de point de vue (141-142) : 
133) Du royaume de Dainville Gérard seul héritier - à la suite du père, Karl, qui vendit tout, perte à laquelle je n'avais jamais songé, considérant uniquement la mienne, bien antérieure - a uniquement conservé ce morceau de mon enfance (...) (Lucot, Frasques, 2001)

134) Je me suis inscrit à un cours de bridge. Mais alors je n'y comprends rien. Le professeur nous explique à chaque fois, et d'une semaine sur l'autre, j'ai tout oublié. (Mréjen, Eau sauvage, 2004)

135) nous tombons sur le cabas de ma grand-mère jeté à terre comme une flaque de vomi, tout a éclaté à l'intérieur, (Guibert, Mausolée des amants, 2001)

136) Ici, tout va très bien à part ma sciatique qui n'en finit pas et m'empêche de marcher. (Mréjen, Eau sauvage, 2004)

137) même que notre séparation cherchait tout à coup à nous réunir et qu'elle y parvenait devant ce bouquet et dans ce silence et pendant ces quelques secondes trafiquées tout devint réellement beau et harmonieux et rouge et blanc et orange entre nous (Bouillier, Invité mystère, 2004)

138) (...) et même ce mois-là que la sonde Ulysse atteignit enfin le Soleil après avoir parcouru des centaines de millions de kilomètres depuis la Terre et ce jour d'octobre 1990 où tout avait finalement redémarré pour moi et pour ainsi dire décollé entre nous (Bouillier, Invité mystère, 2004)

139) Mon père a dit: "Il s'est tué en asséchant un canal. Du haut d'une digue en terre. Tu vides avec une motopompe. On l'a retrouvé, tout tournait toujours, Puech enroulé autour de l'arbre de cardan de la pompe. 》(Bon, Mécanique, 2001)

140) Je tourne le problème dans ma tête, j'ai des angoisses... Je n'en dors pas la nuit. Et voilà qu'au dernier moment, souvent, tout s'éclaircit. (Mréjen, Eau sauvage, 2004)

141) Dans toute notre histoire, sauf la première fois, c'est moi qui ai tout fait. (Ernaux, Se perdre, 2001) puis je juge normale son ignorance, tout aussi déterminée que le cycle des vents et des molécules : pour ce bourgeois, tout va bien. (Lucot, Frasques, 2001)

142) ses traits se détendirent et elle eut un petit sourire comme si tout s'éclairait à présent pour elle et rentrait heureusement dans l'ordre (Bouillier, Invité mystère, 2004)

Partant de là, nous appuyant sur la description des SN en tout élaborée par Kleiber (à par.), nous ferons l'hypothèse que :

i) Tout $t_{\mathrm{P}}$ en tant que marqueur de totalité "établit que le domaine est entièrement parcouru " (Kleiber),

ii) L'hétérogénéité nécessitée par tout en général provient, dans le cas de tout $\mathrm{p}_{\mathrm{p}}$ de l'absence de référence à une quelconque classe et du fait que le pronom désigne un ensemble ouvert, d'occurrences non catégorisées, possiblement éparses dans l'espaceltemps,

iii) le caractère factuel et virtuel des occurrences de tout $\mathrm{p}_{\mathrm{p}}$ empêche le parcours d'occurrence à occurrence ce qui fait que, comme le dit Kleiber, à propos de tout N, le « parcours <est> en quelque sorte simulé ou seulement lancé, mais non effectué véritablement puisque les distinctions qualitatives et identitaires ne sont qu'évoquées et ne sont pas précisément prises en compte ": le trait massif de tout ${ }_{\mathrm{P}}$ a pour effet de gommer ces distinctions et d'accentuer la virtualité du parcours,

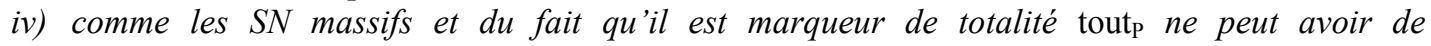
" conditionnement » ou de bornes qu'extrinsèques,

v) les seules bornes extrinsèques disponibles en l'occurrence pour l'interprétation lui sont conférées par la "situation" d'énonciation dans laquelle il s'inscrit (via les temps grammaticaux et les $S N$ adverbiaux aidant à son ancrage).

Dans cette hypothèse, tout $_{P}$ se revèle parfaitement adapté aux contextes " génériques », dont les caractéristiques verbales sont congruentes avec son caractère d'ensemble ouvert, hétérogène et massif. Inversement, la dimension ouverte et virtuelle de son ensemble de référence contrevient, en quelque sorte, aux situations spatio-temporellement ou/ou « épistémiquement » bornées.

De là vient que tout $_{P}$, dans ce cas, provoque un effet d'exagération ou implique, comme le dit Andersson (op. cit., 198) quelque chose de plus ${ }^{17}$ qui fait qu'un énoncé comme (143) « sonne faux » - et donne à entendre que la totalité visée n'est « une vue de l'esprit » :

143) J'ai tout aimé, tout vu, tout su (C ${ }^{\text {sse }}$ de Noailles, Le Bidois, 244). 


\section{Références bibliographiques}

Andersson, S. (1954): Etude sur la syntaxe et la sémantique du mot français Tout, Lund, Carl Bloms Boktryckeri.

Anscombre J.-C. (2006) Tout, n'importe lequel, chaque : quelques remarques, Corblin F., Ferrando S., Kupferman L. (éds), Indéfini et prédication, Paris, PUS, 431-448.

Corblin F. (1995) Les formes de reprise dans le discours. Anaphores et chaînes de référence, Rennes, PU.

De Mulder W. \& Schnedecker C. (2001) Les référents évolutifs entre philosophie et linguistique, Paris, Klincksieck.

Flaux N. (à par.) Les pronoms indéfinis en français : une classe à redéfinir ?, Travaux de linguistique.

Galmiche M. (1988) Massif/comptable : de l'un à l'autre et inversement, David J. \& Kleiber G. (éds), Termes massifs et termes comptables, Paris, Klincksieck, 63-77.

Galmiche M. \& Kleiber G. (1996) Sur les noms abstraits, N. Flaux et al. (éds), (éds) Les noms abstraits. Histoire et Théorie. Lille. PUL, 23-40.

Kleiber G. (1994) Nominales. Essais de sémantique référentielle, Paris, A. Colin.

Kleiber G. (1998) Tout et ses domaines : sur la structure tout + déterminant + N, Englebert A. et al. (éds), La ligne claire. De la linguistique à la grammaire, Bruxelles, Duculot, 87-98.

Kleiber G. (à par.) La quantification universelle en trio : tous les, chaque et tout, Kleiber G. \& Schnedecker C., La quantification et ses domaines, Caen, PUC.

Kleiber G. \& Martin R. (1977) La quantification universelle en français, Semantikos 2, 19-36.

Lammert M. (2006) Sémantique et cognition : les noms collectifs. Thèse de doctorat NR, Strasbourg 2.

Lammert M. (à par.) Sur la quantification totale. $17^{\text {ièmes }}$ Rencontres linguistiques en pays rhénan, Octobre 2007.

Le Bidois G. \& Le Bidois R. (1967) Syntaxe du français moderne, Paris, Ed. A. Picard.

Paillard D. (2001) Tout en français vs vskaji en russe, Blanco X. et al. (éds) Détermination et formalisation, Amsterdam, J. Benjamins, 275-290.

Schnedecker C. (à par. a) De au total à total : comment un quantificateur adverbial en arriver à reformuler et à conclure ?, Colloque Voies de la reformulation (contraintes, stratégies, objectifs), Rennes 19-20 mai 2006 (M.-C. Le Bot \& M. Schuwer, org.).

Schnedecker C. (à par. b), En tout et au total: des adverbiaux jumeaux ?, in Mélanges offerts en l'honneur de H. Nølke, Berne, P. Lang.

Tamba I. (1994) Un puzzle sémantique : le couplage des relations de tout à partie et de partie à tout, Le gré des langues 7, 64-85.

${ }^{1} C f$. une étude à paraitre de Flaux sur la caractérisation des pronoms dits indéfinis.

${ }^{2}$ Même s'il est principalement employé au singulier : Frantext n'atteste que 5 occurrences où tout est précédé de les, 31 de des, 3 de plusieurs et 1 de divers.

${ }^{3}$ Sans décompte précis, c'est ce qui ressort de notre corpus.

${ }^{4}$ Selon des modalités variées : reprise stricte comme dans (53) mais aussi via les pronoms 0 , le, y, ou des $\mathrm{SN}$ mettant en évidence le caractère composite (mélange, composition, pâte, etc.). La place nous manque pour détailler cette question. $C f$. De Mulder \& Schnedecker (2001).

${ }^{5}$ Sur ce distingo, voir Corblin 1995.

${ }^{6}$ Nous utilisons ce terme de manière parfaitement empirique et intuitive sans préjuger pour l'instant de sa correction (compte tenu des $\mathrm{N}$ dont le français dispose pour désigner ce genre d'entités : collection, somme, intégralité, totalité, groupe, etc. étudiés en partie par Lammert, 2006).

${ }^{7}$ Encore que les choses ne soient pas si simples ce qu'illustrent: J'ai commandé un steak. Je n'avais pas faim mais j'ai tout mangé. Je me suis acheté un livre de 300 pages pour agrémenter mon voyage en train. J'ai tout lu en trois heures. 
ISBN 978-2-7598-0358-3, Paris, 2008, Institut de Linguistique Française

Sémantique

DOI $10.1051 / \mathrm{cmlf08} 190$

${ }^{8}$ Les avis divergent sur ce dernier énoncé...

${ }^{9}$ Ce qui les distingue des $\mathrm{N}$ collectifs au sens strict, caractérisés par l'homogénéité des parties entre elles.

${ }^{10}$ Même si, comme nous l'a suggéré G. Kleiber, rien ne permet de statuer sur tout ( $\mathrm{N}$ ou pronom) dans tout ou partie.

${ }^{11}$ Même si, comme nous le fait observer un des rapporteurs, le partitif est envisageable dans certains emplois comptables comme p.e. dans Il a vu de ses amis.

${ }^{12}$ Sauf dans des emplois comme Tout a une fin qui n'équivaut pas toutefois à une fin est une partie de tout

13 «La notion de contrôle, dit l'auteur (art. cit.: 75), (...) semble incompatible avec la représentation séparée des parties et du tout qu'impliquent les relations d'englobant/englobé et de composant/composé».

${ }^{14}$ Il est question dans la citation de tout déterminant.

${ }^{15}$ Plutôt extensionnelles, comme nous le précise un des rapporteurs.

${ }^{16}$ La cooccurrence de tout et des adverbiaux toujours et souvent le distingue de tout $+N$.

${ }^{17}$ La remarque d'Andersson s'applique aux emplois résomptifs de tout $_{P}$ mais vaut tout aussi bien, selon nous, pour les emplois « absolus ». 